Fourth International Conference on Sustainable Construction Materials and Technologies http://www.claisse.info/Proceedings.htm

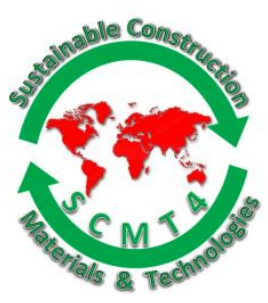

SCMT4

Las Vegas, USA, August 7-11, 2016

\title{
Urban Highway Life Cycle Assessment and Data Collection Methodology
}

\author{
Eleanor Bloom ${ }^{\text {1a }}$, Gregory J. Horstmeier ${ }^{1 \mathrm{~b}}$, Angela Pakes Ahlman ${ }^{\text {cc }}$, and Tuncer B. Edil ${ }^{1 \mathrm{~d}}$ \\ ${ }^{1}$ Recycled Material Resource Center, University of Wisconsin-Madison, 1415 Engineering Drive, \\ Madison,WI, USA. ${ }^{1 a}$ Email: <efbloom@wisc.edu>, ${ }^{1 b}$ Email: <ghorstmeier@wisc.edu>, ${ }^{1 c} P . E .$, LEED \\ AP, Email: <angela.pakes@wisc.edu>, ${ }^{l d}$ Ph.D., P.E., D. GE, Distinguished Member, ASCE, Email: \\ $<$ tbedil@wisc.edu>.
}

\begin{abstract}
Life cycle assessment (LCA) of using recycled materials in roadways is currently not well-understood or documented. A research team from the Recycled Materials Resource Center (RMRC) analyzed the use of recycled materials in the reconstruction of the Beltline Highway in Madison, Wisconsin, to assess quantitatively and accurately the environmental and economic benefits of sustainable road construction. Recycled materials used in the reconstruction and expansion of an urban, 2.4 kilometer (1.5 mile) stretch of the highway included fly ash, recycled concrete aggregate, recycled asphalt shingles and recycled asphalt pavement. In addition to assessing the benefits of recycled material use, the RMRC used this project to better determine a methodology for gathering input data for a LCA and a life cycle cost analysis (LCCA) using PaLATE program. PaLATE is a spreadsheet-based LCA/LCCA tool was used to conduct the analyses for the Beltline case study. In past case studies looking at LCA/LCCA of recycled materials use, the RMRC was unable to utilize LCA and LCCA technology without significant assumptions for the program's inputs. The Beltline project offered a unique opportunity to develop a method for data collection that reduces assumptions for the analyses. The actual design with recycled material use was compared to a reference design using only virgin materials. Results show that the actual design reduced environmental impacts over the highway's lifetime in all criteria, including energy use (13\% reduction), water consumption ( $12 \%$ reduction) and $\mathrm{CO}_{2}$ emissions (13\% reduction). The LCCA showed overall economic savings of approximately $\$ 250,000$ (10\% savings). These results suggest that incorporating recycled materials improves the sustainability of road construction. The knowledge gained from this project will set the precedent for future analyses of department of transportation projects that use recycled materials.
\end{abstract}

\section{INTRODUCTION}

The construction of sustainable roads has become increasingly popular because of global climate change and rising costs of virgin materials. A goal of the Recycled Materials Resource Center (RMRC) is to validate the environmental and economic benefits of incorporating recycled materials into road construction. As a case study, the RMRC quantitatively analyzed recycled materials in the reconstruction and expansion of a 2.4 kilometer (1.5 mile) stretch of the eastbound Beltline Highway (US 12/14 west of 
the Verona Rd. interchange, US 12/14/18/151 east of the Verona Rd. interchange) from Whitney Way to Seminole Highway in Madison, Wisconsin (Figure 1).

In previous case studies, post construction data led to issues, such as over-generalization of mix designs and sourcing, averaging market prices for materials, and inability for real-time data collection. This project demonstrates real-time data collection, a better methodology for gathering input data for the life cycle assessment (LCA) and life cycle cost analysis (LCCA) tool in order to reduce assumptions. The knowledge gained from this project will set the precedent for environmental and economic analyses of larger Wisconsin Department of Transportation (WisDOT) projects that use recycled materials.

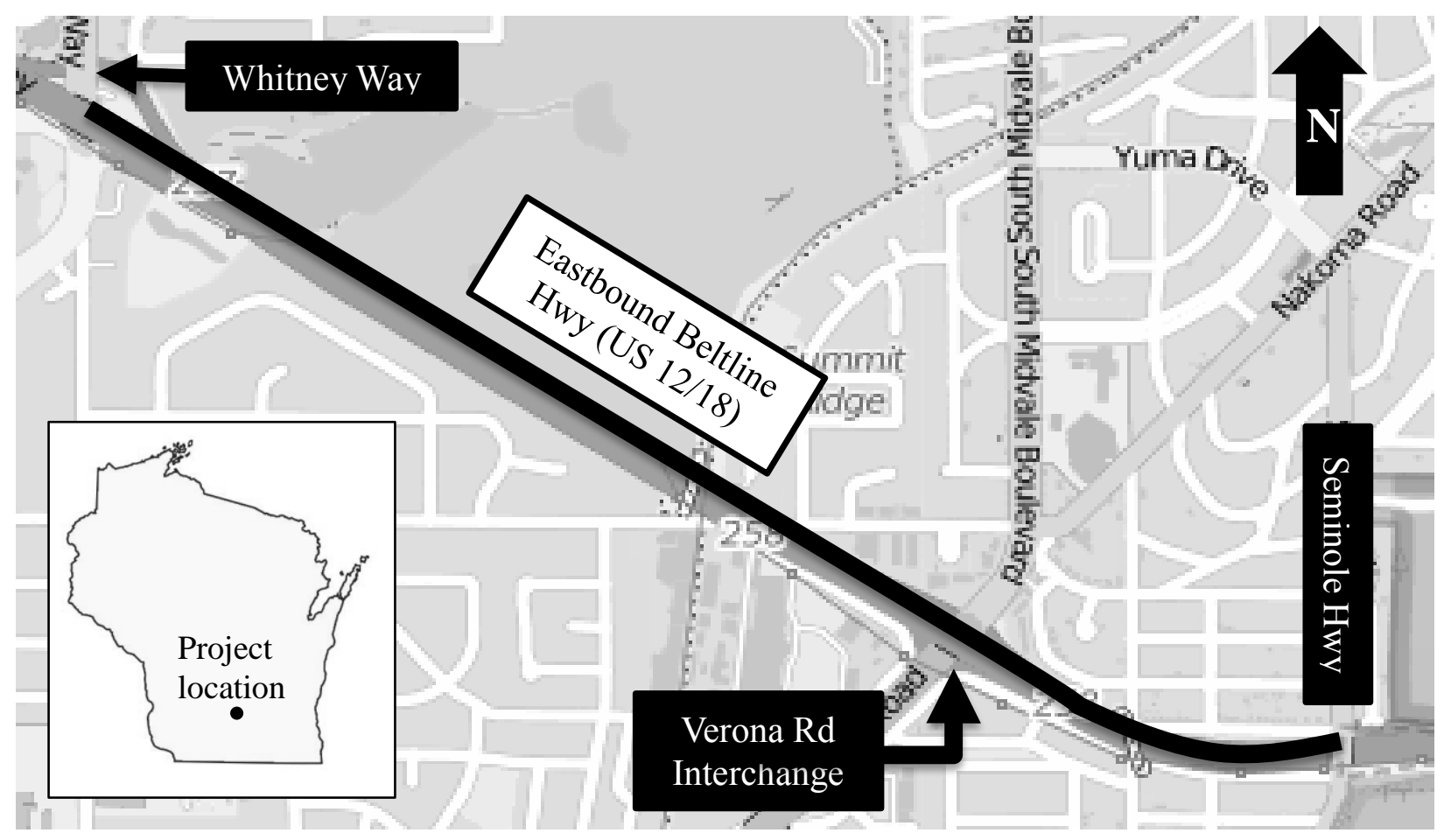

Figure 1. Site Location

\section{BACKGROUND}

Beltline Reconstruction. The Beltline Highway is a multi-lane, urban, and major arterial highway used by a substantial number of local and regional travelers in the Madison area. Fed by numerous local roads, county roads, and other major routes in southwest Wisconsin, the Beltline is a crucial route for both trucks and passenger vehicles (WisDOT, 2015a). Although the Beltline from Verona Rd. to Seminole Highway is part of the Wisconsin Backbone System (US 151) from Dubuque, IA, to Fond du Lac, WI, the area in this study is the only section that is not a four-lane expressway/freeway (FHWA and WisDOT, 2011).

Key reasons for the Beltline's expansion are safety and population growth. According to WisDOT, numerous sections of the Beltline have crash rates higher than the state average for similar highways. The population of Dane County is estimated to increase by approximately 150,000 residents by 2035, with vehicle traffic on Verona Rd. increasing to 52,900 to 68,800 vehicles per day by the year 2030 (Increased mobility is vital to the efficiency of Beltline travelers. 
This RMRC analysis focuses on the first part of stage one of this project: eastbound Beltline Highway reconstruction from Whitney Way to Seminole Highway. The reconstruction involves expanding to three lanes in each direction, at times with an auxiliary lane, and installing a Single Point Urban Interchange (SPUI) spanning Verona Road (Strand Associates, 2014). Incorporating a multitude of ramps, the SPUI design improves traffic capacity by executing additional turning opportunities either on the overpass or underpass (FHWA, 2010). The existing hot mix asphalt (HMA) surface pavement was replaced with 28$\mathrm{cm}$ concrete pavement, at times over an asphaltic base. Six ramps were updated and four were added. Construction began in spring of 2014 on Verona Road and in fall of 2014 on the Beltline. Construction is expected to end in late 2015 or early 2016 for eastbound lanes and in fall 2016 for westbound lanes (WisDOT, 2015b).

The typical existing pavement structure varies for the 2.4-km stretch of this study, but was generally comprised of the following layers (Strand Associates, 2014).

- $\quad 7.6-15 \mathrm{~cm}$ HMA pavement

- $23 \mathrm{~cm}$ reinforced concrete pavement

- $7.6 \mathrm{~cm}$ asphaltic stabilized base course (varies)

- $\quad 15-30 \mathrm{~cm}$ crushed aggregate base course

The reconstruction design chosen for the $2.4-\mathrm{km}$ stretch is generally comprised of the following layers:

- $28 \mathrm{~cm}$ reinforced concrete pavement

- $15 \mathrm{~cm}$ base aggregate dense $3.2 \mathrm{~cm}$

- $41 \mathrm{~cm}$ select crushed material (SCM)

Urban vs. Rural Construction. This study highlighted the differences between the uses of recycled material in urban versus rural construction. The Beltline construction is categorized as urban principal arterial (FHWA and WisDOT, 2011). Although urban construction has time and area constraints not seen in rural construction, urban settings generally have greater access to resources and suppliers. For example, the number of quarries, construction equipment, and laborers increases as one goes from a rural to an urban area.

Time constraints were enacted by the City of Madison so that major construction did not occur during peak travel times. Thus, fast drying concrete mixes, which exclude fly ash, were required in some areas. Mixes omitting fly ash include Type C, High Early Strength (HES), and Super High Early Strength (SHES). Space constraints are prevalent in urban settings, decreasing storage area flanking the highway for recycled asphalt pavement (RAP) and recycled concrete aggregate (RCA). Thus, recycled material from the existing road may either be trucked offsite or immediately placed for embankment/fill or base course. Once removed from the site, the contractor can choose to store, sell, or landfill the material. According to the contractor, almost all onsite RAP and RCA were immediately used for the new Beltline Highway.

Life Cycle Assessments. LCA and LCCA were conducted to quantify the environmental and economic benefits gained from using recycled materials in reconstruction. LCA quantifies environmental impacts over the lifetime of a product by using a meticulous evaluation methodology. LCCA outlines cost comparisons among design alternatives, denoting economic benefits. For this study, two designs were analyzed: Actual and Reference. The Actual design uses the real amounts of recycled material included in the Beltline reconstruction. In the Reference design, recycled material quantities were replaced with 
virgin materials, i.e. the Reference design is $100 \%$ virgin materials. By this method, percent savings from the actual use of material can be determined.

\section{ANALYSIS AND METHODS}

PaLATE. PaLATE is both a spreadsheet LCA and LCCA program designed by the Consortium on Green Design and Manufacturing from the University of California, Berkeley, and commissioned by the RMRC. PaLATE assesses the environmental and economic effects of pavement and road construction. Users input the initial design, initial construction materials and transportation, maintenance materials and transportation, equipment use, and cost for a road. PaLATE calculates environmental impacts in three stages of the roads construction: material processing, materials transportation, and installation processes/equipment. Environmental outputs include (Horvath, 2007):

- Energy consumption (GJ)

- Water consumption $(\mathrm{kg})$

- $\mathrm{CO}_{2}$ emissions $(\mathrm{kg})$

- $\mathrm{NO}_{\mathrm{x}}$ emissions $(\mathrm{kg})$

- $\mathrm{PM}_{10}$ emissions $(\mathrm{kg})$

- $\mathrm{SO}_{2}$ emissions $(\mathrm{kg})$

- CO emissions (kg)

- Leachate information (mercury, lead, Resource Conservation and Recovery Act (RCRA) hazardous waste generated, and both cancerous and non-cancerous human toxicity potential (HTP))

Data Collection Methodology. One goal of this study was to determine a data collection methodology to save time and reduce assumptions in future LCAs and LCCAs of road construction projects. Because realtime data was not collected in previous studies, estimates of recycled quantities were made based on finished road plans. This method was avoided in the Beltline study, as actual used quantities of materials could be tracked and recorded. PaLATE also contained some outdated information that the RMRC has since updated.

Data Collection - As the sole sponsoring agency, WisDOT selected the Beltline reconstruction project as the first point of reference for this study. The WisDOT project manager helped define the scope of work and provided key assumptions for the project. As research progressed, RMRC researchers contacted contractor and sub-contractor representatives. Quantities and procedures were clarified. Key site-specific WisDOT and sub-contractor files, including Item Record Account (IRA) spreadsheets, Quality Management Plan (QMP) specifications, concrete and HMA pavement mix designs, site plans, and bid item lists, were accessed for information on materials. Such files aided in tracking materials, specifically material type, volume, tonnage, unit cost, equipment/processes for installation, and transportation distance. Weigh tickets were also critical in data collection because they specified the material, its origin, and its quantity. Additionally, WisDOT provided a maintenance schedule over the 50-year lifetime of the road, including material quantities for the rehabilitation processes. Relevant maintenance, as determined by the WisDOT Pavement Type Selection Report, includes repair and grind in years 25 and 33, and repair and overlay in year 41 (WisDOT, 2015c). The same methodology used for initial construction was implemented for the maintenance analysis. All future maintenance costs have been brought to present value.

Recycled Pavement Calculations - Omitted from weigh tickets were pavements recycled in situ, such as RAP and RCA used as base course or embankment/fill. To account for these un-weighed materials, the 
site plans were used to calculate the tonnage of RAP and RCA recycled from the existing road. After calculating the volume of the existing pavement and concrete based on plan dimensions, density conversions were used to determine tonnage of RAP and RCA. The amount of base aggregate was tracked for QMP purposes. The quantity designated for embankment/fill was estimated as the difference between the total RAP and RCA, and the RAP and RCA used for base course.

This estimate was deemed valid because the contractor stated a) in the Beltline construction, almost all RAP and RCA was immediately used for the new highway and b) rebar was the majority of the reinforcement in existing concrete, while significantly smaller amounts of mesh reinforcement were found. Mesh causes separation difficulties, so concrete not easily separated was not recycled in the new highway.

Data Refinement and Input - Because raw material quantities are required for the PaLATE analysis, data was compiled into spreadsheets that categorized bid items by their raw materials. For instance, typical Portland Cement Concrete (PCC) consists of cement, fly ash, aggregates, and water. For each raw material, a transportation distance, transportation vehicle, and unit price were determined. A hypothetical Reference design of all virgin materials was compared to an Actual design incorporating the quantities of recycled materials. Using PaLATE, LCA determined the environmental impact, and LCCA determined the economic savings.

Assumptions. The assumptions made while performing the LCA are as follows:

- Although only the eastbound portion of the construction was analyzed, minor westbound construction started in fall 2014, carrying into April 2015. Thus, all material quantities from the start of construction until April 1, 2015 were divided in half to estimate materials for eastbound work alone. These adjusted quantities were likewise used for the LCCA.

- Any maintenance material quantities provided by WisDOT were assumed to be for the entire road. Therefore, these quantities were divided in half to estimate materials for eastbound work alone.

- Existing road dimensions were used to calculate volume of RAP and RCA. Average widths were used when ranges were provided.

- Supplier locations were obtained via weigh tickets and QMP reports. Transportation distances were determined from the material origin to either the plant locations or the Beltline/Verona Rd. intersection.

- Unless otherwise stated, the assumed transportation vehicles were dump trucks, with the exception of cement trucks for cement/fly ash.

- Virgin material (Reference) was substituted ton-for-ton for recycled material (Actual). In reality, different dimensions or quantities of virgin material may be required to construct the Reference design road to meet the structural support requirement.

- Recycled aggregate from offsite quarries was deemed RCA.

- Only bid items that contained or had the potential to contain recycled material were analyzed. This included pavements, concrete structures, base aggregates, and subbases such as SCM.

- Only the total volume of the PCC surface material was provided. The amount of individual material was calculated from the proportions in the PCC mixes used by the pavement supply company. The same process was used for HMA pavement raw material calculations.

- Table 1 includes the recycled materials and their assumed virgin counterparts. 
Table 1. Recycled Materials and Virgin Material Counterparts

\begin{tabular}{|c|c|}
\hline Recycled Material & Virgin Material Counterpart \\
\hline RAP in base course or embankment/fill & Gravel aggregate \\
\hline RCA in base course or embankment/fill & Gravel aggregate \\
\hline Fly Ash & Portland Cement \\
\hline Blast Furnace Slag & Portland Cement \\
\hline RAP in HMA (binder) & Asphalt cement \\
\hline RAP in HMA (aggregate) & Virgin aggregate in HMA \\
\hline RAS & Virgin aggregate in HMA \\
\hline
\end{tabular}

\section{RESULTS}

Life Cycle Assessment. The results of the Beltline LCA for all construction completed by the end 2015, are listed in Table 2. In general, reductions were seen in all PaLATE categories. The percent reduction was calculated by the difference in impact of the Actual and Reference divided by the Reference's impact. The greatest reduction is seen in $\mathrm{PM}_{10}$ at $20 \%$. Because more recycled material was used with a smaller transportation distance, less vehicles and equipment were used on and off site, resulting in fewer particulate emissions.

Table 2. PaLATE Results of Beltline Analysis Completed in October 2015.

\begin{tabular}{|c|c|c|c|c|c|c|c|c|}
\hline & Energy (GJ) & \multicolumn{2}{|c|}{ Water (kg) } & $\mathrm{CO}_{2}(\mathrm{Mg})$ & \multicolumn{2}{|c|}{$\mathrm{NO}_{\mathbf{x}}(\mathbf{k g})$} & $\mathbf{P M}_{10}(\mathrm{~kg})$ & $\mathrm{SO}_{2}(\mathrm{~kg})$ \\
\hline Actual & 140,587 & \multicolumn{2}{|c|}{41,259} & 9,731 & \multicolumn{2}{|c|}{96,566} & 34,150 & 390,030 \\
\hline Reference & 161,744 & \multicolumn{2}{|c|}{46,639} & 11,095 & \multicolumn{2}{|c|}{98,697} & 44,957 & 393,749 \\
\hline \multirow[t]{2}{*}{ Reduction } & $13 \%$ & \multicolumn{2}{|c|}{$12 \%$} & $12 \%$ & \multicolumn{2}{|c|}{$2 \%$} & $24 \%$ & $1 \%$ \\
\hline & CO (kg) & Hg (g) & $\mathbf{P b}(\mathrm{g})$ & $\begin{array}{r}\text { RC } \\
\text { Hazardo } \\
(\mathrm{k}\end{array}$ & Waste & HT & $\begin{array}{l}\text { P cancer } \\
(\mathrm{kg})\end{array}$ & $\begin{array}{c}\text { HTP non } \\
\text { cancer }(\mathrm{Mg})\end{array}$ \\
\hline Actual & 37,848 & 162 & 9,032 & 786 & & & 0,643 & 29,383 \\
\hline Reference & 38,660 & 171 & 9,826 & 858 & & & 9,556 & 32,839 \\
\hline Reduction & $2 \%$ & $5 \%$ & $8 \%$ & 8 & & & $-11 \%$ & $11 \%$ \\
\hline
\end{tabular}

The next largest reductions are in energy (13\%), water (12\%), and $\mathrm{CO}_{2}$ emissions (12\%). Energy, water, and $\mathrm{CO}_{2}$ impacts largely stem from the materials' production. Mining and grading virgin aggregated is a more resource intensive processes than milling and grinding existing pavement. Similarly, milling asphalt pavement and grinding recycled shingles to use in HMA is a far less intensive process than producing 
asphalt cement or aggregates for the mix. Because fly ash is a by-product, any energy, water or emissions associated with it production are not included. Compared to the production of concrete, using fly ash allows for significant impact reductions.

Important reductions to note are those for HTP cancer (-11\%) and non-cancer (11\%). HTP is an index for the potential harm of a unit of chemical released into the environment (Hertwich et al, 2001). It is based on the chemical's inherent toxicity and potential dose, and can be evaluated in terms of carcinogens and non-carcinogens. In PaLATE, a 11\% reduction in non-cancerous HTP indicates that by substituting recycled material, less non-cancerous toxins have the potential to be released into and harm the environment. HTP cancerous saw an increased impact, as indicated by its $-11 \%$ "reduction". The potential for harmful carcinogens to be released from the use of recycled pavements and by-products is slightly greater than that of virgin gravel, rock, and cement.

Additional reductions are seen in material transportation. Any recycled material transported from off-site locations have relatively similar transportation impacts compared with the conventional material. However, the pavements recycled onsite and used directly in the new construction's base have minimal transportation impact. This leads to a significant overall reduction in any environmental impact associated with transportation. Material processing and equipment, or constructing the actual roadway, saw slight increases in most environmental impact categories due to the use of recycled material. Although the HMA and concrete pavements had the same impact whether or not recycled materials were used, the aggregate and gravel placing and compaction impact was different. In PaLATE's analysis, the density difference between recycled aggregate and virgin aggregate lead to a slightly more intensive base course placing and compaction process. For most categories, this increase in impact is negated by the reductions saved during the materials' production and transportation.

Life Cycle Cost Analysis. The costs used in the cost analysis came from a variety of sources. To calculate the savings, the cost for a recycled material was compared to the cost for an equivalent virgin material (e.g. fly ash vs. cement). A summary of the unit costs are listed in Table 3. The unit price for both fly ash and cement were provided by the Wisconsin Concrete Pavement Association (WCPA). The average savings of $\$ 30 /$ ton are based on historical Wisconsin averages.. The prices for RCA, both from existing pavement onsite and hauled in from offsite, were also provided by WAPA. The savings were found by comparing the reduced prices of RCA to WisDOT's average bid item price for base aggregate, $\$ 10 /$ ton (WisDOT, 2015d). This led to savings of $\$ 4.50 /$ ton and $\$ 1.00 /$ ton for RCA from onsite and offsite, respectively.

The cost for RAP recycled onsite into base aggregate was also found from the WisDOT average bid item price list. On average, WisDOT pays $\$ 6.00 /$ ton for salvaged asphaltic pavement milling. This leads to savings compared to conventional base aggregate ( $\$ 10 /$ ton) of $\$ 4.00 /$ ton. Cost savings for RAP in HMA pavement were provided by the Wisconsin Asphalt Pavement Association (WAPA). The average cost for HMA mix with no RAP in Wisconsin is $\$ 49.47 /$ ton, while a mix with approximately $16 \%$ HMA costs $\$ 43.75 /$ ton.. This leads to a savings of $\$ 5.72 /$ ton of mix that uses RAP as asphalt cement or aggregate. For the Beltline, RAS was also used as an aggregate in some HMA mixes. According to the National Asphalt Pavement Association (NAPA), RAS for pavement construction purposes costs basically nothing. Therefore, all savings by not using conventional aggregate are realized. Using the WisDOT average bid item cost for aggregate, this equates to saving \$10/ton of RAS.

LCCA savings for the initial construction are estimated to be $\$ 35,255$ from RAP in HMA, $\$ 1,259$ from the use of RAS in HMA, \$133,014 from the use of both on and off site RAP and RAS as base aggregate and SCM, and $\$ 48,950$ from the substitution of fly ash for cement. This equates to total initial construction savings of over $\$ 219,478$ reducing the cost by $9 \%$. Savings were also estimated for future 
maintenance materials over 50 years, which will include concrete with fly ash and HMA with RAP and RAS. The total maintenance savings at present value are $\$ 27,196$. This equates to a grand total saved over the lifetime of the project of nearly $\$ 250,000$, a $9 \%$ reduction in cost compared to the reference design.

Table 3. Materials and Unit Costs for 2.4-km of Highway Construction

\begin{tabular}{|c|c|c|c|}
\hline Category Analyzed & Material & Unit Cost & Source \\
\hline \multirow{3}{*}{\begin{tabular}{c} 
Concrete \\
\cline { 2 - 4 } Base Aggregate (including \\
SCM)
\end{tabular}} & Fly Ash & $\$ 75.00$ & WCPA \\
\cline { 2 - 4 } & Cement & $\$ 105.00$ & WCPA \\
\cline { 2 - 4 } & RAP onsite & $\$ 6.00$ & WisDOT \\
\cline { 2 - 4 } & RCA onsite & $\$ 5.50$ & WCPA \\
\hline \multirow{3}{*}{$\begin{array}{c}\text { RCA offsite } \\
\text { HMA }\end{array}$} & Mirgin Base Aggregate & $\$ 10.00$ & WCPA \\
\cline { 2 - 4 } & Mix without RAP & $\$ 49.47$ & WisDOT \\
\cline { 2 - 4 } & RAS & $\$ 42.75$ & WAPA \\
\cline { 2 - 4 } & Aggregate in HMA & $\$ 10.00$ & WAPA \\
\cline { 2 - 4 } & & & WisDOT \\
\hline
\end{tabular}

\section{CONCLUSION}

The methodology determined by this case study has proven to be an effective means of not only tracking recycled materials in road construction, but also quantitatively analyzing their environmental and economic benefits. Real-time data collection reduces significant assumptions, thus adding credibility to results. Another key aspect of this study was updating the analysis program, PaLATE, and cost data.

Based on a PaLATE analysis of the eastbound Beltline reconstruction, the use of recycled materials reduces the environmental impact of the highway over its lifetime. In a life cycle analysis comparison of a Reference highway design using no recycled material and the Actual design with the recycled materials, the Actual design improved the environmental impact of the road by all criteria. Additionally, life cycle cost analysis showed promising overall economic reductions of $9 \%$. These results suggest that incorporating recycled materials vastly improves the sustainability of road construction. The knowledge gained from this project will set the precedent for future material tracking and analysis for department of transportation projects. A continuation of this analysis may include the westbound Beltline reconstruction, including a results comparison to this eastbound Beltline investigation.

\section{ACKNOWLEDGEMENT}

Funding for this project was provided by the Wisconsin Department of Transportation (WisDOT) through the Construction Materials Support Center (CMSC) at the University of Wisconsin-Madison. The authors thank Steve Krebs and Chris Fredrick from WisDOT and Gary Whited at CMSC. Additional acknowledgements are to contractors on the project including Joe Jirsa, Mary Gherke, Greg Courter, and Kevin Kaufman. Additionally, thank you to Kevin McMullen of WCPA, Brandan Strand of WAPA, and Kent Hansen of NAPA. 


\section{REFERENCES}

Federal Highway Administration. (2010). "Alternative Intersections/Interchanges: Information Report (AIIR)." Federal Highway Administration Research and Technology, Publication Number: FHWAHRT-09-060, < http://www.fhwa.dot.gov/publications/research/safety/09060/009.cfm> (Aug. 12, 2015).

Federal Highway Administration (FHWA) and State of Wisconsin Department of Transportation (WisDOT). (2011). "United States Highway 18/151 (Verona Road) Final Environmental Impact Statement." Federal Highway Administration, Report No. FHWA-WIS-EIS-03-02-F, Madison, WI.

Hertwich, E. G., Mateles, S. F., Pease, W. S., and McKone, T. E. (2001). "Human toxicity potentials for life-cycle assessments and toxics release inventory risk screening." Environmental Toxicology and Chemistry, 20(4), 928-939.

Horvath, A. (2007). "PaLATE: Pavement Life-cycle Assessment Tool for Environmental and Economic Effects." Consortium on Green Design and Manufacturing, University of California, Berkeley, $<$ http://www.ce.berkeley.edu/ horvath/palate.html> (Aug. 12, 2015).

Strand Associates. (2014). "Plan of Proposed Improvement: USH 18." State of Wisconsin Department of Transportation, Project ID: 1206-07-84/85, Madison, WI.

Wisconsin Department of Transportation (WisDOT). (2015a). "Madison Beltline Study." WisDOT Projects by Region, < http://wisconsindot.gov/Pages/projects/byregion/sw/madisonbeltline/default.aspx> (June 2015).

Wisconsin Department of Transportation (WisDOT). (2015b). "Verona Road (US 18/151) Project." 511 Wisconsin Construction Projects, http://projects.511wi.gov/veronard/ (June 2015).

Wisconsin Department of Transportation (WisDOT). (2015c). "Chapter 14: Pavement." Facilities Development Manual, <http://wisconsindot.gov/Pages/doing-bus/eng-consultants/cnsltrsrces/rdwy/fdm.aspx > (Dec. 9, 2015).

Wisconsin Department of Transportation (WisDOT). (2015d). "Wisconsin Department of Transportation - Average Unit Price List.” http://wisconsindot.gov/hcciDocs/contracting-info/average-unit-price.pdf (Dec. 9, 2015). 